\title{
Classification methods, Deep Learning Architecture, Data source and Challenges in Detection of Breast Cancer
}

\author{
Nalini Sampath, N. K. Srinath
}

\begin{abstract}
Different types of cancer can be prevented, screened for and/or detected and treated at an early stage. According to recent statistics breast cancer has a mortality rate of 12.7 per one lakh women. Mutation of genes at an abnormal rate leads to cancer. Changes in the size, color, texture and constant pain are the initial symptoms of breast cancer. A person presented with these symptoms requires breast cancer screening which would help in the diagnosis. Early detection can help health care professionals to start with the treatment, thereby reducing the mortality rate. Recent advances in breast cancer detection have proven to aid both medical professional and patients in making health care decisions. In this paper image acquisition technique, classification techniques, deep learning models and data sets available are highlighted.
\end{abstract}

Keywords: classification, dataset, deep learning, imaging modality, transfer learning.

\section{INTRODUCTION}

Breast Cancer was first reported during 3000-2500 B.C.E. Hippocrates, the father of medicine described the various stages of breast cancer and mentioned that cancer is caused due to excess of black bile .Breast cancer treatment has significantly improved over years and the various milestones are follows: William Halsted in the year 1882 performed radical mastectomy. Until $20^{\text {th }}$ century radical mastectomy remained the standard operation to treat breast cancer until . Radium was used to treat cancer in the year 1898.Radiation therapy started to be given as an additional treatment in addition to surgery since 1937 . Selective estrogen receptor modulators a new class of drug called Tamoxifen acts against cancer and also decreases the risk of the same by blocking the production of estrogen. According to Globocon 2018 1,62.468 new cases have been registered and 87090 deaths have been reported. Urban women are highly prone to develop breast cancer than the rural women he various parameters with the mortality to incidence ratio are 1:22 and 1:60 respectively.

One of the important advantages of computer aided diagnosis is elimination of operator dependency, improve the diagnostic accuracy and provide a beneficial means for cancer detection and classification. When detected at an early stage and treatment is considered to be easier and successful.

Revised Manuscript Received on December 22, 2019.

Mrs. Nalini, Department of Computer Science, Amrita Vishwa Vidyapeethamin, University, Coimbatore, Tamil Nadu, India.

Dr N K Srinath, Professor, Department of Computer Science and Engineering, RV College of Engineering, Bangalore, India.
Researchers continue to explore better methods that will help a large number of people across the globe but more studies are needed for sure. Cancer research can accelerate our progress towards a world without cancer. need for research to categorization of cancer types according to early symptoms will help the researchers to increase comparability of studies in this field and also will provide guidance for health personnel to set up a primary care in low income regions [1]. The automated algorithms in the detection of breast cancer can significantly reduce physician time, Cases that need special attention can be prioritized and that would lead to better outcomes for patients, at much lower costs.

When a person is diagnosed with cancer, doctors will try to find out if it has spread and the severity. Based on the biopsy report the pathologist will be able to identify them as benign or malignant. A benign cancer does not invade to other tissues where as a malignant would. The classification of cancer is important to achieve three important aims i.e diagnosis, prognosis and prediction.

In this paper a detailed review on breast cancer is presented. Section II covers a detailed review on Imaging Modality, Classification Technologies in Breast Cancer Detection, Deep Learning models and the datasets available. Section III discusses the challenges in developing an automated diagnosis system and Section IV a framework for future work is proposed.

\section{REVIEW OF LITERATURE}

\section{A. Imaging Modality}

Mammography remains an effective and gold standard method to detect breast cancer before the lesions become clinically palpable [2].Women with no signs or symptoms of breast cancer can undergo a mammogram screening .Human breast are examined using low dose of X-rays. Masses of cancer and calcium deposits will appear brighter. Calcifications and Ductal Carcinoma In Situ (DCIS) are best diagnosed with mammography The drawbacks of this are it exposes us to radiation and sometimes can find something that is abnormal but that isn't cancer. Cancer can be detected by film and digital mammography and are similar [3-5]. 
Palpable and impalpable abnormalities in the breast can also be detected with Breast Ultrasound can also be done in addition with mammography so that the diagnosis is more accurate supported by the clinical examination[2]. Ultrasound transducer uses high frequency sound waves that are directed into the breast tissues. The reflected sound waves can be detected by the transducer. Asymptomatic women when screened by ultrasound results in unacceptable false positive and false negative outcomes [6], due to which this screening method is mostly not preferred.

Breast Thermography is also called as thermal imaging. It is used by medical practitioners to monitor changes in the breast that could indicate breast cancer. Higher metabolism occurs in cancerous tissues and results in a faster growth of cancer cells [2]. Normal breast tissue is lower in temperature when compared to the cancerous and pre-cancerous tissues. This temperature difference can be used in the detection of breast cancer. Analysis and interpretation of thermograms largely depends on analysts [2].

Magnetic Resonance Imaging is an imaging modality that is applied not only in screening high risk cancer patients for diagnosis but also in staging and follow up on breast cancer. Breast MRI uses intravenous contrast administration (Gadolinium) but not limited by breast density and preferentially detects the higher-grade lesions [7]. MRI might actually detect the more clinically relevant high grade lesions. The low grade DCIS readily picked up by the X ray mammogram may be missed on MRI. On the other hand $10-15 \%$ of DCIS present as non-calcifying DCIS are missed $\mathrm{X}$ ray mammogram but detected on MRI [7].

Positron emission mammography (PEM) plays an important role in the diagnosis and monitoring of malignant tumors [8]. Glucose metabolic activity of the tissues can be monitored by this technique. Normal cells have a decreased glucose metabolism as compared to the malignant tumors. A good contrast between cancerous and normal cells in PET images is visible [2].

Scintimammography or breast-specific gamma imaging (BSGI) is performed to detect cancerous tissues where radioactive chemical is injected into the blood, and the breast examined is examined using a special camera [9]. Whole body is exposed to radiation in this method and hence not a safe screening method to be used every year.

Electrical impedance imaging (EIT), the conductivity of breast cancer cells is different from that of normal cells. Small electrodes are taped to the skin of the breast and electric current is passed through. As radiation is not used it is considered to be a safer screening method .This method can be used in addition to mammography. This test can be used to help classify tumors found on mammograms [9].

\section{B. Classification Methods in Breast Cancer Diagnosis}

Machine Learning based and Convolution Neural Network based have proven successful in developing an end to end automated system for breast cancer diagnosis. Recent research carried out the models such as k- Nearest Neighbors, Support Vector Machine, Random Forest and Naïve Bayes models and their results are reported tabulated.

k-Nearest Neighbors method can be applied for problems based on both classification and regression. The evaluation parameter for any algorithm is based on three important aspects namely time for execution, predictive power and whether it is easy to interpret the output. K-NN works based on the principle that similar data points stay closer in the same surroundings[22]. $\mathrm{k}-\mathrm{NN}$ in comparison with other algorithms fairs well across all the above mentioned parameters. Sharma et al. [14] in their work proved k-NN showed an accuracy of $95.90 \%$.

Support Vector Machine - a machine learning model used to classify by minimizing generalization error. SVM is well suited for classification of complex datasets and it does not work well for large datasets [9] .SVM model consists of different kernel. Linear or polynomial kernel can be chosen. According to results the SVM with kernel classifier has a better classification accuracy and its performance is better than k-Means based classification methodologies [10]. Classification accuracy in SVM multi kernel model for malignant, benign and normal cells is $90.8 \%, 90.3 \%$ and 90.03 respectively.

Performance of C4.5, Naïve Bayes, Support Vector Machine (SVM) and K- Nearest Neighbor (KNN) was compared in various parameters in order to find the best classifier and Support Vector Machine turned out to be the most accurate with an accuracy of 96.99\%[11]. Highest accuracy of SVM classifier was $97.9 \%$ reported in [12].

A supervised learning algorithm creates an ensemble of decision tress and the system is trained using the bagging method [14]. This is the approach followed in Random Forest Classifier. Literature review based on random forest classifier has proven to have the best values for precision and recall scores as $99 \%$. Sharma et al. [14] in their work proved that the classification of breast cancer into benign and malignant showed an accuracy of $94.74 \%$. This method can be used for classification problems but it does not perform well for regression problem as it prediction values are not continuous in nature.

Naive Bayes classifier a collection of algorithms based on the principle that every pair of features being classified is independent of each other. Sharma et al. [14] in their work proved that using Naïve Bayes classifier showed an accuracy of $94.74 \%$. The only disadvantage of this method is that it does not deal with regression problems [14]. The other models like k- NN and Random Forest can handle both Performance of the classification methods was reported in [12] is tabulated in TABLE I [12]. 
TABLE I. PERFORMANCE OF THE CLASSIFIERS.

\begin{tabular}{|c|c|c|c|c|}
\hline $\begin{array}{c}\text { Evaluation } \\
\text { criteria }\end{array}$ & \multicolumn{4}{|c|}{ Classifiers } \\
\hline $\begin{array}{c}\text { Time to } \\
\text { build model } \\
(\mathrm{s})\end{array}$ & 0 & 0.08 & 0.28 & 0.01 \\
\hline $\begin{array}{c}\text { Correctly } \\
\text { classified } \\
\text { instances }\end{array}$ & 547 & 557 & 546 & 527 \\
\hline $\begin{array}{c}\text { Incorrectly } \\
\text { classified } \\
\text { instance }\end{array}$ & 22 & 12 & 23 & 42 \\
\hline $\begin{array}{c}\text { Accuracy } \\
(\%)\end{array}$ & 96.1 & 97.9 & 96 & 92.6 \\
\hline TP Rate & 0,961 & 0,979 & 0,960 & 0,926 \\
\hline FP Rate & 0,046 & 0,034 & 0,055 & 0,086 \\
\hline Recall & 0,961 & 0,979 & 0,960 & 0,926 \\
\hline Precision & 0,961 & 0,979 & 0,960 & 0,926 \\
\hline
\end{tabular}

Genetic algorithm is used in combination with various other machine learning methods and it could be considered as an optimizer. This method could be best suited for feature selection which in turn can improve the other parameters such as accuracy, specificity and sensitivity. Genetic Algorithm was applied in [17] to detect micro calcifications which are one of the deciding factors in the detection of breast cancer .In [17] an algorithm was proposed that resulted in Az value of 0.9.Application of Neural Network model for classification have produced $96.57 \%$ of accuracy value with 0.987 of AUC and is recorded as an excellent value [17].

\section{Deep Learning Models.}

Recent work based on CNN models are aimed to help reading and analyzing the breast cancer images more accurately by the radiologists. They are composed of multiple processing layers to retrieve features from raw data with multilevel representations [15].Convolution layer, full -connection layers, pooling layers in addition to the input and output layers are the main components in the design of a Convolution Neural Network architecture. Such models are capable of incorporating handcrafted features and this increases the classification performance [16].

VGG Net is a model where the error rate is under $10 \%$. The time taken to train a model using VGG Net is less compared to other model. Primarily it was developed to reduce the number of parameters in the convolution layers. Multiple variants of VGG Net differ in the number of layers in the network. The most often used architecture are VGG16 and VGG19. VGG16 has a total of 138 million parameters. Simonyan et al. in [18] showed through evaluation of network a significant improvement on the prior-art configuration by increasing depth with $(3 \times 3)$ convolution filters, was achieved by pushing the depth to 16-19 weight layers.

Alex Net consists of 5 convolutional layers and 3 fully connected layers. The activation function it uses is ReLU. The advantage of using ReLU is does not suffer from Vanishing Gradient (VG )problem. It uses an additional drop out layer to address the overfitting problem..It has a of total of 62 million trainable variables. A patch and image-wise accuracy of $75.73 \%$ and $81.25 \%$ was acheived on the validation set and image-wise accuracy of $57 \%$ by Nawaz et al. in [19]. It has a uniform architecture with lots of filters and classified the images into four classes.

Google Net architecture is well known deep learning model that consists of many layers .It consists of 22 layers deep and the number of parameters is reduced to 4 million. Small convolutions done on the inception module helped in the reduction of parameters. This is an important feature of this model . Saikia et al. in [20] used different architectures and concluded that an accuracy of $96.25 \%$ was achieved by fine tuning GoogLeNet-V3..

\section{Data source}

Wisconsin Breast Cancer Diagnosis (WBCD) is a benchmark dataset that consists of mammogram images .Most often used data set with Machine learning methods. The accuracy is high ranging between $96.36 \%$ and $99.90 \%$ [23] when implemented by Machine learning algorithms. 699 instances are available and almost ten real valued can be computed. Ten real-valued features are computed for each cell nucleus such as radius, texture perimeter, area, smoothness, compactness, concavity, concave points, symmetry and fractal dimension are used in the classification process. One of the disadvantages of this model is under sampling by which important information may be lost.

Breast Cancer Digital Repository provides a reference to explore computer aided detection. Mammography and ultrasound images, clinical history, lesion segmentation and selected pre- computed image based descriptors of 1734 patients are a part of this data set. Clinical and Image based features can be extracted from this bench marking dataset which contains mass and micro calcification based images [24]. It is a promising database but still in its development phase. The images are of high resolution and the masses don't greatly vary in shape.

Digital Database for Screening Mammography (DDSM) is the largest public database which contains mammogram images .Around 3061 mammograms from 1597 cases are annotated based on region of interest[27]. These images are taken from different views which include cranio-caudal (CC) and medio-lateral oblique (MLO) views for most of the screened breasts [31].The images are not compressed and require additional decompression to be done. Precise mass boundary is not outlined in DDSM and only a general location is available [25].

INbreast database is an annotated dataset with a total of 115 cases. There are seven types of annotations: asymmetry, calcification, cluster, mass, distortion, speculated region and pectoral muscle [29].Positive patches are extracted from the mammograms which show masses [31].

Mammographic Image Analysis Society (MIAS) database contains 322 images which are digitized and are of low resolution. Pre processing is an essential step when using this dataset as the images are noisy .. The digitized films are of size $2.3 \mathrm{~GB} 8 \mathrm{~mm}$ (ExaByte) tape. 
MIAS database images are compressed to a 200 micron pixel edge.All the images are of the available in $1024 \times 1024[26]$. This database images contain normal and abnormal images. The abnormal ones are marked by radiologist.

Image Retrieval in Medical Application is limited in size and lacks variations in shape which is one of the disadvantage .High resolution is an added feature and the lesions are segmented with greater accuracy.

\section{RESULTS}

The various challenges in implementing a solution for automated diagnosis are discussed below.

Most of the datasets that are publicly available are often incomplete. They lack clinical features and provide very little description about the image. Example of a large but non annotated dataset is BreakHis which contains histopathological images. Choosing an annotated dataset may result in a smaller dataset like BreCAHAD. It is a tedious task to train a dataset using deep architectures. Annotation of images requires expertise. An imbalanced dataset when used for classification purpose will bias prediction towards normal cases resulting in an increase of false negatives. Hwang et al. [28] proposed a self-transfer learning framework that can be effectively used for a different target domain. Another worth mentioning challenge is memory constraint and computational requirements.

\section{CONCLUSION}

Hematoxylin and eosin stained histopathological breast cancer images can be considered as a dataset. The images in this dataset are in different magnification factors. The whole images can be cropped into multiple patches. Each of the patches can then be given as an input to the chosen deep learning model. Once the relevant features are learned by the network, it can be tested. The patch wise images can be fused by choosing an appropriate fusing algorithm. This step can be performed on the complete training dataset. Depending on the class labels chosen they can be classified as benign or malignant.

Transfer learning can be further used on a different target domain or different dataset. This can ensure better results in terms of precision and recall. Diagnosis of breast cancer with true positive results can be taken up to the next stage to assess the pathological grading for further treatment.

\section{REFERENCES}

1. Ott, J.J., Ullrich, A. and Miller, A.B., 2009. The importance of early symptom recognition in the context of early detection and cancer survival. European Journal of Cancer, 45(16), pp.2743-2748.

2. Sree, S.V., Ng, E.Y.K., Acharya, R.U. and Faust, O., 2011. Breast imaging: a survey. World journal of clinical oncology, 2(4), p.171.

3. Skaane, P., Hofvind, S. and Skjennald, A., 2007. Randomized trial of screen-film versus full-field digital mammography with soft-copy reading in population-based screening program: follow-up and final results of Oslo II study. Radiology, 244(3), pp.708-717.

4. Pisano, E.D., Gatsonis, C., Hendrick, E., Yaffe, M., Baum, J.K., Acharyya, S., Conant, E.F., Fajardo, L.L., Bassett, L., D'Orsi, C. and Jong, R., 2005. Diagnostic performance of digital versus film mammography for breast-cancer screening. New England Journal of Medicine, 353(17), pp.1773-1783.

5. Pisano, E.D., Hendrick, R.E., Yaffe, M.J., Baum, J.K., Acharyya, S., Cormack, J.B., Hanna, L.A., Conant, E.F., Fajardo, L.L., Bassett, L.W. and D'Orsi, C.J., 2008. Diagnostic accuracy of digital versus film mammography: exploratory analysis of selected population subgroups in DMIST. Radiology, 246(2), pp.376-383.

6. Teh, W. and Wilson, A.R.M., 1998. The role of ultrasound in breast cancer screening. A consensus statement by the European Group for Breast Cancer Screening. European journal of cancer, 34(4), pp.449-450.

7. Yu, Q., Huang, K., Zhu, Y., Chen, X. and Meng, W., 2019. Preliminary results of computer-aided diagnosis for magnetic resonance imaging of solid breast lesions. Breast cancer research and treatment, pp.1-8.

8. Jadvar, H., Alavi, A. and Gambhir, S.S., 2009. 18F-FDG uptake in lung, breast, and colon cancers: molecular biology correlates and disease characterization. Journal of Nuclear Medicine, 50(11), pp.1820-1827.

https://www.cancer.org

9. Chanda, P.B. and Sarkar, S.K., 2018, December. Detection And Classification Technique Of Breast Cancer Using Multi Kernal SVM Classifier Approach. In 2018 IEEE Applied Signal Processing Conference (ASPCON) (pp. 320-325). IEEE.

10. Bashir, S., Qamar, U. and Khan, F.H., 2015. Heterogeneous classifiers fusion for dynamic breast cancer diagnosis using weighted vote based ensemble. Quality \& Quantity, 49(5), pp.2061-2076.

11. Khourdifi, Y. and Bahaj, M., 2018, December. Applying Best Machine Learning Algorithms for Breast Cancer Prediction and Classification. In 2018 International Conference on Electronics, Control, Optimization and Computer Science (ICECOCS) (pp. 1-5). IEEE.

12. Akila, A. and Padma, M.R., 2019. Breast Cancer Tumor Categorization using Logistic Regression, Decision Tree and Random Forest Classification Techniques. International Journal of Research in Arts and Science, 5(Special Issue Holistic Research Perspectives [Volume 4]), pp.282-289.

13. Sharma, S., Aggarwal, A. and Choudhury, T., 2018, December. Breast Cancer Detection Using Machine Learning Algorithms. In 2018 International Conference on Computational Techniques, Electronics and Mechanical Systems (CTEMS) (pp. 114-118). IEEE.

14. LeCun, Y., Bengio, Y. and Hinton, G., 2015. Deep learning. nature, 521(7553), pp.436-444.

15. 16 . Zou, L., Yu, S., Meng, T., Zhang, Z., Liang, X. and Xie, Y., 2019. A Technical Review of Convolutional Neural Network-Based Mammographic Breast Cancer Diagnosis. Computational and mathematical methods in medicine, 2019.

16. Karnan, M. and Thangavel, K., 2007. Automatic detection of the breast border and nipple position on digital mammograms using genetic algorithm for asymmetry approach to detection of microcalcifications. Computer methods and programs in biomedicine, 87(1), pp.12-20.

17. Simonyan, K. and Zisserman, A., 2014. Very deep convolutional networks for large-scale image recognition. arXiv preprint arXiv:1409.1556.

18. Nawaz, W., Ahmed, S., Tahir, A. and Khan, H.A., 2018, June. Classification of breast cancer histology images using alexnet. In International Conference Image Analysis and Recognition (pp. 869-876). Springer, Cham

19. Saikia, A.R., Bora, K., Mahanta, L.B. and Das, A.K., 2019. Comparative assessment of $\mathrm{CNN}$ architectures for classification of breast FNAC images. Tissue and Cell, 57, pp.8-14.

20. Silvana, M., 2018, October. Optimization of Neural Network with Genetic Algorithm for Breast Cancer Classification. In 2018 International Conference on Information Technology Systems and Innovation (ICITSI) (pp. 398-403). IEEE.

21. Chougrad, H., Zouaki, H. and Alheyane, O., 2018. Deep convolutional neural networks for breast cancer screening. Computer methods and programs in biomedicine, 157, pp.19-30.

22. Halim, E., Halim, P.P. and Hebrard, M., 2018, September. Artificial Intelligent Models for Breast Cancer Early Detection. In 2018 International Conference on Information Management and Technology (ICIMTech) (pp. 517-521). IEEE.

23. www.bcdr.edu

24. . Lee, R.S., Gimenez, F., Hoogi, A., Miyake, K.K., Gorovoy, M. and Rubin, D.L., 2017. A curated mammography data set for use in computer-aided detection and diagnosis research. Scientific data, 4, p.170177. 
25. http://www.mammoimage.org/databases/

26. Shen, L., Margolies, L.R., Rothstein, J.H., Fluder, E., McBride, R. and Sieh, W., 2019. Deep Learning to Improve Breast Cancer Detection on Screening Mammography. Scientific reports, 9.

27. Hwang, S. and Kim, H.E., 2016, October. Self-transfer learning for weakly supervised lesion localization. In International Conference on Medical Image Computing and Computer-Assisted Intervention (pp. 239-246). Springer, Cham.

28. Moreira, I.C., Amaral, I., Domingues, I., Cardoso, A., Cardoso, M.J. and Cardoso, J.S., 2012. Inbreast: toward a full-field digital mammographic database. Academic radiology, 19(2), pp.236-248.

29. Araújo, T., Aresta, G., Castro, E., Rouco, J., Aguiar, P., Eloy, C., Polónia, A. and Campilho, A., 2017. Classification of breast cancer histology images using convolutional neural networks. PloS one, 12(6), p.e0177544.

30. 31 . Agarwal, R., Diaz, O., Lladó, X., Yap, M.H. and Martí, R., 2019. Automatic mass detection in mammograms using deep convolutional neural networks. Journal of Medical Imaging, 6(3), p.031409.

\section{AUTHORS PROFILE}

Mrs. Nalini, Sampath is working at Amrita Vishwa Vidyapeethamin Department of Computer Science. She is pursuing research at VTU. Her research area includes Image Processing, Machine learning.

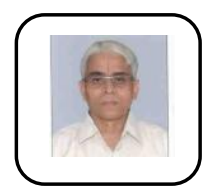

Dr N K Srinath, working as a Professor in the Department of Computer Science and Engineering at RV College of Engineering, Bangalore. He has 32 years of rich experience in the Academics. He has published more than 40 research articles in National and International Journals. He holds CSI membership and an active member in CSI events. His research area includes High performance computing, Image processing. 